\title{
COMMENTARY ON \\ "NONUNIQUE TANGENT MAPS \\ AT ISOLATED SINGULARITIES OF HARMONIC MAPS" BY BRIAN WHITE
}

\author{
WILLIAM P. MINICOZZI II
}

\begin{abstract}
Immediately following the commentary below, this previously published article is reprinted in its entirety: Brian White, "Nonunique tangent maps at isolated singularities of harmonic maps", Bull. Amer. Math. Soc. (N.S.) 26 (1992), no. 1, 123-129.
\end{abstract}

Brian White's 1992 article in the Bulletin of the American Mathematical Society W1 gave an ingenious construction of the first examples of singularities with a continuum of limiting blowups. These examples, which came a decade after the major uniqueness of blow-up results by Allard and Almgren $\mathrm{AA}$ and Leon Simon [S showed the subtlety of the uniqueness question. Two and a half decades later, these problems and techniques remain central to the understanding of singularities in geometry and analysis.

Blow-up analysis. Blow-up analysis is a way to either rule out or, more generally, understand singularities by zooming in to smaller and smaller scales and then analyzing the limit. The simplest example is the following characterization of differentiability:

A function $u: \mathbf{R} \rightarrow \mathbf{R}$ is differentiable at a point $x$ if any sequence of dilations of its graph converges to a line.

For example, if $\gamma \subset \mathbf{R}^{2}$ is the graph $\{(x, u(x)) \mid x \in \mathbf{R}\}$ of $u, u(0)=0$ and $r_{i} \rightarrow \infty$, then the curves $r_{i}(\gamma)$ converge to a line precisely when $u$ is differentiable at $x$. The limiting line is just the tangent line to the graph $\gamma$ at the origin.

A similar blow-up analysis, where one zooms in on a point to get limiting tangent information, is an important tool for understanding singularities in geometry. This method has been very successful in many different geometric problems, including minimal varieties, harmonic maps, and mean curvature flow, among others. In each case, the existence of a limit is guaranteed by a general compactness theorem, and a priori control on the dilates comes from a monotone scale-invariant quantity.

Blow-up analysis for minimal varieties. The prototype for geometric blow-up analysis comes from the work of Federer and Fleming [FF in 1960 on minimal varieties:

Received by the editors April 3, 2018.

2010 Mathematics Subject Classification. Primary 58E12, 58E15, 58E20, 53D44.

The author was partially supported by NSF Grant DMS- 1707270. 
Any sequence of dilates of a minimal variety has a subsequence weakly converging to a minimal cone (the limit is minimal and dilation invariant).

If we blow up at a smooth point, then the limiting minimal cone is just the tangent plane to the variety at that point. At a singular point, however, the limiting minimal cone, the tangent cone, describes the nature of the singularity. Moreover, there are powerful techniques from geometric measure theory that control the size and structure of the singular set if one can analyze the possible tangent cones.

Blow-up analysis for harmonic maps. Schoen and Uhlenbeck SU] developed a blow-up theory for harmonic maps in the early 1980s. In this case, the blow-up process gives a limiting tangent map. The tangent map is homogeneous of degree zero (this plays the analogous role of dilation invariance for minimal cones). The tangent map is constant precisely when the harmonic map itself is smooth. At a singular point, the tangent map contains information about the singularity and, in some cases, can be used to rule singularities all together (for example, when the target has nonpositive curvature; see [SU] p. 310]). Perhaps the simplest nonsmooth example is the map $x \rightarrow \frac{x}{|x|}$ from the 3 -ball to the 2 -sphere.

There are also monotonicity formulas for the parabolic versions of these problems - mean curvature flow $[\mathrm{H}]$ and the harmonic map heat flow [St. In these cases, the blowup is a tangent flow that is invariant under parabolic dilations in space and time.

Uniqueness of blowups. The blow-up process raises an obvious question: Is it possible that a different subsequence of dilates converges to a different limit blowup? As White explains for minimal varieties in his 1998 article [W2] on Fred Almgren:

In other words, a minimal variety looked at under a microscope will resemble a cone, but under higher magnification, it might (as far as anyone knows) resemble a completely different cone. Whether this ever happens remains perhaps the most fundamental open question about singularities of minimal varieties.

The general question of uniqueness of blowups for minimal varieties remains open, though there has been substantial progress over the last five decades. One of the early uniqueness results was the 1973 theorem of Jean Taylor T] for a special class of minimal varieties in three dimensions (cf. W3 for surfaces in higher codimension).

Taylor used the epiperimetric inequality, following ideas of Reifenberg from 1964, to prove a rate of convergence and uniqueness. She then used the uniqueness (and the ideas in its proof) to get a very complete description of the possible singularities: the singular set must be a differentiable curve. Much of the later work on uniqueness has a similar theme, where the uniqueness is the starting point for analyzing the finer structure of the singular set.

General uniqueness results. The early results on uniqueness were tailored to a specific problem, but the early 1980s saw two powerful approaches that have since been applied to a wide variety of settings. These methods were developed by Allard and Almgren $[\mathrm{AA}$ and Simon $[\mathrm{S}$. To explain these methods, note that the blowup process gives that the set of possible tangent cones form a continuous family. Thus, uniqueness would be clear at an isolated minimal cone. Unfortunately, at 
the very least, any cone sits in a family consisting of all Euclidean rotations of the cone, so they will never be isolated. Allard and Almgren instead assumed an integrability condition, asserting that every infinitesimal one-parameter family of variations actually came from differentiating an actual family. Simon's approach did not require this integrability, but rather required some analyticity and then appealed to powerful results from real algebraic geometry.

Nonuniqueness. As we will see below, White's 1992 examples of nonuniqueness of blowups were for harmonic maps. Namely, he constructed a smooth 4-manifold $N$ and a harmonic map from the 3-ball to $N$ with an isolated singularity at 0 and a continuous family of (distinct) tangent maps at the singularity. This example shows that Simon's celebrated uniqueness theorem for harmonic maps to analytic spaces fails without the analyticity, raising many interesting questions and illustrating the subtlety, and difficulty, of the uniqueness of blowups in general.

Recent developments. There is a great deal of activity on uniqueness of blowups and structural consequences for the singular set. We refer to [DSS, GW], Ha], [RT], and references therein, for results related to minimal varieties and harmonic maps and [CM1, CM2, GK], W4, W5 for results on mean curvature flow.

\section{REFERENCES}

[AA] William K. Allard and Frederick J. Almgren Jr., On the radial behavior of minimal surfaces and the uniqueness of their tangent cones, Ann. of Math. (2) 113 (1981), no. 2, 215-265, DOI 10.2307/2006984. MR607893

[CM1] Tobias Holck Colding and William P. Minicozzi II, Uniqueness of blowups and Eojasiewicz inequalities, Ann. of Math. (2) 182 (2015), no. 1, 221-285, DOI 10.4007/annals.2015.182.1.5. MR 3374960

[CM2] Tobias Holck Colding and William P. Minicozzi II, Level set method for motion by mean curvature, Notices Amer. Math. Soc. 63 (2016), no. 10, 1148-1153, DOI 10.1090/noti1439. MR 3559343

[DSS] Camillo De Lellis, Emanuele Spadaro, and Luca Spolaor, Uniqueness of tangent cones for two-dimensional almost-minimizing currents, Comm. Pure Appl. Math. 70 (2017), no. 7, 1402-1421, DOI 10.1002/cpa.21690. MR.3666570

[FF] Herbert Federer and Wendell H. Fleming, Normal and integral currents, Ann. of Math. (2) 72 (1960), 458-520, DOI 10.2307/1970227. MR0123260

[GK] Zhou Gang and Dan Knopf, Universality in mean curvature flow neckpinches, Duke Math. J. 164 (2015), no. 12, 2341-2406, DOI 10.1215/00127094-3146175. MR.3397388

[GW] Robert Gulliver and Brian White, The rate of convergence of a harmonic map at a singular point, Math. Ann. 283 (1989), no. 4, 539-549, DOI 10.1007/BF01442853. MR990588

[Ha] Robert M. Hardt, Singularities of harmonic maps, Bull. Amer. Math. Soc. (N.S.) 34 (1997), no. 1, 15-34, DOI 10.1090/S0273-0979-97-00692-7. MR1397098

$[\mathrm{H}] \quad$ Gerhard Huisken, Asymptotic behavior for singularities of the mean curvature flow, J. Differential Geom. 31 (1990), no. 1, 285-299. MR.1030675

[RT] Tristan Rivière and Gang Tian, The singular set of 1-1 integral currents, Ann. of Math. (2) 169 (2009), no. 3, 741-794, DOI 10.4007/annals.2009.169.741. MR2480617

[SU] Richard Schoen and Karen Uhlenbeck, A regularity theory for harmonic maps, J. Differential Geom. 17 (1982), no. 2, 307-335. MR664498

[S] Leon Simon, Asymptotics for a class of nonlinear evolution equations, with applications to geometric problems, Ann. of Math. (2) 118 (1983), no. 3, 525-571, DOI 10.2307/2006981. MR 727703

[St] Michael Struwe, On the evolution of harmonic maps in higher dimensions, J. Differential Geom. 28 (1988), no. 3, 485-502. MR965226

[T] Jean E. Taylor, Regularity of the singular sets of two-dimensional area-minimizing flat chains modulo 3 in $R^{3}$, Invent. Math. 22 (1973), 119-159, DOI 10.1007/BF01392299. MR0333903 
[W1] Brian White, Nonunique tangent maps at isolated singularities of harmonic maps, Bull. Amer. Math. Soc. (N.S.) 26 (1992), no. 1, 125-129, DOI 10.1090/S0273-0979-1992-00254-9. MR 1108901

[W2] Brian White, The mathematics of F. J. Almgren, Jr, J. Geom. Anal. 8 (1998), no. 5, 681702, DOI 10.1007/BF02922665. Dedicated to the memory of Fred Almgren. MR.1731057

[W3] Brian White, Tangent cones to two-dimensional area-minimizing integral currents are unique, Duke Math. J. 50 (1983), no. 1, 143-160, DOI 10.1215/S0012-7094-83-05005-6. MR700134

[W4] Brian White, The size of the singular set in mean curvature flow of mean-convex sets, J. Amer. Math. Soc. 13 (2000), no. 3, 665-695, DOI 10.1090/S0894-0347-00-00338-6. MR 1758759

[W5] Brian White, The nature of singularities in mean curvature flow of mean-convex sets, J. Amer. Math. Soc. 16 (2003), no. 1, 123-138, DOI 10.1090/S0894-0347-02-00406-X. MR 1937202

Department of Mathematics, Massachusetts Institute of Technology, Cambridge, MASSACHUSETTS

Email address: minicozz@math.mit.edu 